\title{
MisPRONOUNCED LEXICAL ITEMS IN POLISH ENGLISH OF ADVANCED LEARNERS
}

\author{
JOLANTA SZPYRA-KOZLOWSKA \\ Maria Curie-Skłodowska University
}

\begin{abstract}
The paper is a continuation of the author's earlier studies in which she argues that it is the mispronunciation of whole words due to their incorrect phonological storage in the learners' phonetic memory that is more detrimental to successful communication via English than an inaccurate production of individual segments and suprasegmentals. Consequently, phonetically difficult words deserve to be thoroughly investigated and pedagogically prioritized.

The present study is a report on an experiment in which 20 English Department students, all advanced learners of English, were recorded having been asked to read a list of diagnostic sentences containing 80 words known to be problematic for Poles in terms of their pronunciation. This has been done in order to isolate and examine the major error types, to establish a hierarchy of difficulty among 8 sources of pronunciation errors, to compare the obtained results with the most common error types made by intermediate learners and to juxtapose the participants' subjective evaluation of the phonetic difficulty of words with their actual phonetic performance. The final goal is to draw pedagogical implications for the phonetic training of advanced students of English.
\end{abstract}

\section{Introduction}

A striking feature of foreign-accented English, including Polish-English, is a frequent occurrence of the so-called local errors, i.e. words stored in the learners' phonetic memory in an incorrect phonological shape. In a number of studies (Szpyra-Kozłowska 2011, Szpyra-Kozłowska and Stasiak 2010, Szpyra-Kozłowska in press) I argue that the use of such items is more detrimental to successful communication via English than inaccurately produced segments and suprasegmentals. In Szpyra-Kozłowska (in press) I present experimental evidence that local errors significantly decrease Polish learners' comprehensibility and intelligibility, create the impression of a heavy foreign accent and are irritating for native English listeners. Consequently, Szpyra-Kozłowska and Stasiak (2010) conclude that a shift is needed in phonetic instruction from the focus on sounds, sound contrasts and prosodies to the focus on the pronunciation of problematic words. To achieve this goal, however, a deeper insight is required into what types of items are phonetically difficult for learners of different L1 background and various levels of language proficiency. Szpyra-Kozłowska (2011) attempts to examine this issue in relation to intermediate Polish learners and identifies eight major sources of word pronunciation errors. 
The present paper undertakes the problem of mispronounced words in the speech of advanced Polish learners of English. It is a report on an experiment in which 20 English Department students of Maria Curie-Skłodowska University in Lublin, Poland, were recorded having been asked to read a list of diagnostic sentences containing 80 words known to be problematic for Poles in terms of their pronunciation. This has been done in order

- to isolate and examine the major types of phonetically difficult words;

- to establish a hierarchy of difficulty among 8 chief sources of pronunciation errors in the speech of advanced learners;

- to compare the obtained results with those of intermediate learners;

- to examine the experimental results with the predictions of the PDI;

- to juxtapose the participants' subjective evaluation of the phonetic difficulty of words with their actual phonetic performance;

- to draw pedagogical implications for the phonetic training of advanced students of English.

It is hoped that although the study is carried out in the Polish context, many of the observations made here will be relevant for other types of foreign-accented English.

\section{Sources of word mispronunciations}

Many sources of word pronunciation errors commonly made by Polish learners are wellknown and have been identified by previous research.

In this context Sobkowiak's (1999) work on the Phonetic Difficulty Index (PDI) should be pointed out as a valuable attempt to deal systematically with phonetically difficult words in Polish English. PDI (p. 214) "is a global numerical measure of the phonetic difficulty of the given English lexical item for Polish learners," meant to be included in machine-readable EFL dictionaries and thus having mainly lexicographic applications. It contains phonetic difficulty ratings of English words carried out by the author on the basis of his observations of Polish learners' pronunciation problems. The current list of error sources (personal communication) includes 61 issues which can, however, be grouped into more general categories. Thus, the largest set (26) concerns spelling-related problems while the next largest group (24) involves problems with the pronunciation of individual sounds and combinations of sounds (e.g. vowel hiatus, consonant clusters). A prominent position is also occupied by stress-related problems (5). The remaining error sources concern the incorrect application of Polish phonological rules (such as Word-Final Obstruent Devoicing and Voice Assimilation) to English, word length (more than 5 syllables) and several others.

It should be added that Sobkowiak's list and his PDI are of general nature and do not specify the relationship between the degree of words' phonetic difficulty and the learners' level of English proficiency. This is a serious drawback of this proposal since what is difficult for beginners might be fairly trivial for more advanced students. In other words, the phonetic difficulty of English words should be examined in relation to their proficiency level. 
Taking this fact into account, in a recent study (Szpyra-Kozłowska 2011) I examined the sources of phonetic difficulty of English words in intermediate learners' speech. The following eight major types of issues have been isolated:

1. Spelling-related problems.

2. Phonetic 'false friends.'

3. Stress-related problems.

4. Pronunciation of consonant clusters involving interdentals.

5. Pronunciation of long words.

6. Pronunciation of words containing several liquids.

7. Pronunciation of words containing sequences of high front vowels.

8. Pronunciation of words with morphological alternations in related forms.

9.

Since the types of problems listed above will be subject to experimental verification, some explanation of these issues is in order.

Spelling-related difficulties result from two kinds of interference. The first one involves interference from Polish spelling-to-pronunciation conventions incorrectly applied to English words. Thus, typical examples comprise silent letters pronounced in such items as $\langle\mathrm{t}\rangle$ in nestle and $\langle\mathrm{b}\rangle$ in tomb. Another problem stems from incorrect overgeneralizations of English letter-to-sound rules, for example, interpreting the digraph <ea> as the vowel [i:] in steak (as in meat, leaf, teach) or <ace> as [eIs] in surface and palace (as in face, lace). As Polish learners have more access to written rather than to spoken English, spelling exerts a powerful effect on Polish English pronunciation.

Phonetic 'false friends' are numerous lexical items which occur in both languages in an identical or a similar orthographic form, but with different pronunciation. In the majority of cases they are cognates, e.g. E chaos / P chaos, borrowings from English, e.g. E model / P model or just accidental look-alikes, e.g. E gnat / P gnat 'bone.' A large group of such words are proper nouns which appear in both languages in rather different phonetic shapes, e.g. Nepal-E [nI'po:1] / P ['nepal] and Sidney - E ['sIdnI] / P [s'idnej]. Similar or identical spelling suggests to the learners that their pronunciation must be similar as well.

For speakers of languages with fixed stress, such as Polish, learning the intricacies of the English stress system with its irregularities and exceptions is a genuine challenge. Thus, while Polish learners typically employ Polish penultimate stress to English words, e.g. 'Japan, in'dustry, demon'strated, they frequently stress also other syllables, i.e. ultimate, e.g. e'ffort, fe'male and antepenultimate, e.g. 'successful, 'computer, ar'bitrary (for a more detailed discussion of stress errors in Polish English see Waniek-Klimczak 2002).

The interdental fricatives, which are absent in Polish, belong to the most difficult sounds for many foreign learners. The degree of difficulty increases when they occur in combination with other consonants. Intermediate learners who participated in our previous experiment listed the following difficult words, all of which contain consonantal clusters with interdentals: three throw birthday maths healthy sixth

The next source of difficulty is the length of words. Longer words are problematic for the learners because of a variety of factors to be controlled: the placement of stress, 
the articulation of many different new sounds and complex sound sequences. The question that arises concerns the actual length of words which makes their pronunciation difficult. Below we list some examples, taken from Szpyra-Kozłowska (2011), supplied by intermediate learners as difficult because of their length,

(a) trisyllables: excitement, adventure, Australia, picturesque

(b) quadrisyllables: relaxation, astonishing, surprisingly

(c) quintisyllables: encyclopaedia, occasionally, exaggeration

According to these data, words marked as problematic because of their length contain three syllables or more. For intermediate learners the longer a word, the more difficult it is to pronounce.

One of the most interesting results of our study involving intermediate learners (Szpyra-Kozłowska 2011) was the discovery that the presence of several liquids, i.e. rhotics and laterals, contributes to the considerable pronunciation difficulty of a word. Here are some examples supplied by the participants: appropriate library regularly particularly rarely burglary

Many such items, apart from articulatory difficulty, are problematic because of their spelling since $\langle\mathrm{r}\rangle$, appearing in the word-final and preconsonantal position, is a silent letter in nonrhotic accents such as RP, generally taught in Poland. Since learners are often confused as to which r's to pronounce, many of them attempt to articulate all these letters, which creates several liquids in a single word.

The collected data include also words regarded as difficult by the respondents due to the fact that morphological alternations take place in the roots they contain. Since in English such changes are often highly irregular and idiosyncratic, this fact contributes to the perceived difficulty of the items in question. Some examples are presented below with related forms provided in parentheses.

(a) society (social), northern (north), southern (south), anxiety (anxious)

(b) can 't (can), variety (various), breathe (breath), wi idth (wide),

In (a) segments subject to consonant alternations are underlined while in (b) vowel alternations are indicated. It is likely that pupils learn first more frequent words given in parentheses and when faced with less common related items, transfer the pronunciation from the former to the latter by analogy or due to preserving paradigm uniformity. The degree of difficulty increases due to the fact in the above forms the alternating segments are spelt in the same way.

The last category of phonetically difficult words for intermediate Polish learners comprises items which contain two (a) or three (b) different high front vowels, i.e. [I] and [i:], e.g.

(a) reading, sleeping, cheating, speedy, greedy, sleepy

(b) believing, receiving, preceding, repeating, 
In such instances they tend to employ some kind of vowel harmony and pronounce two [i:] vowels (or rather its shorter and less tense Polish counterpart [i]).

Yet another problem with [I] is created by the following words: innocent, image, impression, important, industry

In these items the initial vowel is difficult for Polish learners to pronounce and usually replaced with Polish [i]. Apart from the powerful influence of English spelling, another active factor here seems to be a phonotactic constraint of Polish banning in the word initial position the occurrence of the Polish front centralized vowel [y], very close to English [I].

It should be added that, as demonstrated earlier, in many cases more factors than one contribute to the phonetic difficulty of words. For instance, long words are often problematic not only due to their length, but also because of the stress placement or combinations of sounds they contain.

\section{Experimental design}

In October 2010 twenty randomly selected $4^{\text {th }}$ year students of the English Department of Maria Curie-Skłodowska University, Lublin, Poland, all advanced learners of English, took part in the experiment in which they were asked to read aloud a list of sentences (see Appendix 1) containing 80 phonetically difficult words, with 10 items representing each of the 8 types of error sources discussed in the preceding section. The students were then individually recorded. After the recording they were given a short questionnaire to complete (see Appendix 2). They were provided with a list of 24 words which appeared in the diagnostic sentences (with 3 items representing each of the 8 categories) and asked to evaluate the degree of phonetic difficulty they posed for them (easy, medium and difficult to pronounce). They were also requested to select three particularly difficult words and comment on the source of the problem. The recordings were next auditorily assessed by the researcher.

\section{Results and discussion}

\subsection{General results}

The experiment yielded 1600 tokens, of which only $655(41 \%)$ were pronounced correctly and 945 (59\%) incorrectly. Graph 1 shows that the results of individual participants range from $22.6 \%$ of correctly pronounced experimental items by the poorest student to $70 \%$ by the best student in this group. The mean result is $36 \%$. 


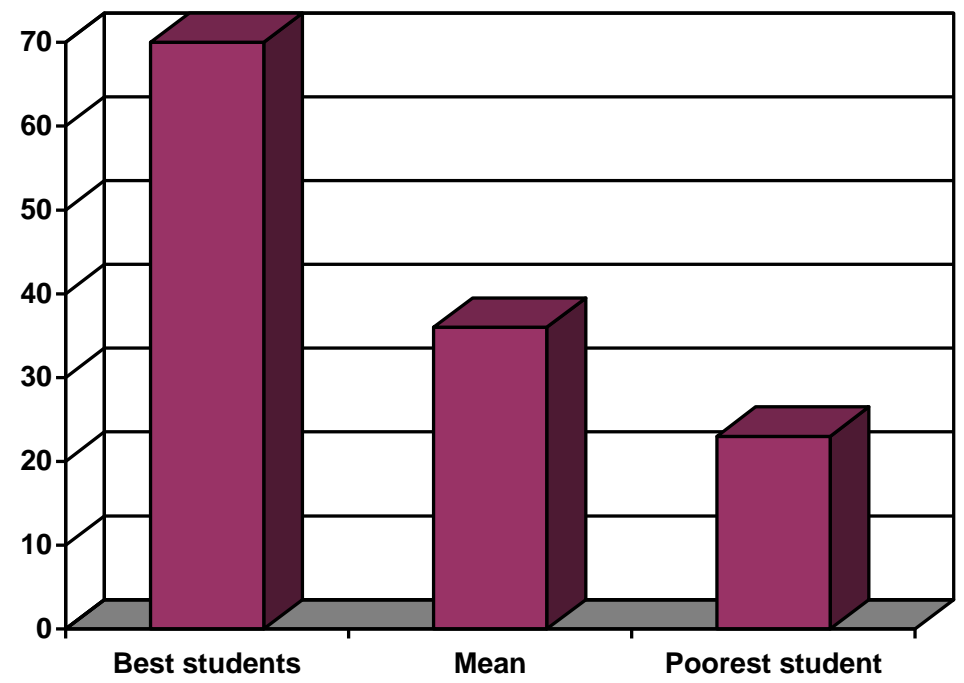

Graph 1. Performance of best and poorest students

The above figures indicate that even for advanced students the experimental items constitute a serious learning problem which has to be approached and remedied.

\subsection{Hierarchy of word pronunciation difficulty factors}

The experimental data allowed us to establish the following hierarchy of difficulty of the 8 factors presented in section 2 for advanced students:

Relatively easy types (over $50 \%$ of correct responses):

Clusters of 'th' and consonants different than $/ \mathrm{s} /-70 \%$

Liquids - 66\% of correctly pronounced tokens (with two words being considerably more difficult, i.e. particularly and regularly - only 7\%)

Stress $-52 \%$ (particularly difficult: caricature)

Long words $-51 \%$ (particularly difficult: artificiality, congratulatory, authoritarian, unintelligibility)

Medium difficulty (25\%-40\% of correct responses)

Spelling - 40\% (particularly difficult: hideous, haven, thoroughly, Graham)

Alternating forms - 33. 5\% (particularly difficult: courteous, advantageous, managerial, infamous)

Considerable difficulty (below $25 \%$ of correct responses):

Phonetic 'false friends' - 24\% (particularly difficult: algebra, gigantic, Disney)

Clusters of 'th' and /s/ - 12\% (particularly difficult: strengths, lengths)

High front vowels $-7.5 \%$ (all difficult) 
The above data require some comments. First of all, within most categories there are words of a different degree of pronunciation difficulty for the participants. Only the set containing sequences of high front vowels is homogeneous in this respect in that all of them proved to be equally problematic. The same is true in the case of clusters of interdentals and other fricatives. Thus, in these two instances we can talk of truly global errors, not restricted to any particular lexical items. In the remaining cases there were both easier and more difficult words, which means that other factors, apart from the ones discussed here, are also relevant here. For example, while the words containing sequences of liquids do not pose any major difficulty for advanced learners, two items, i.e. particularly and regularly are commonly mispronounced by them.

Pedagogical implications of the established hierarchy of word difficulty are obvious. Advanced students should receive additional training in the pronunciation of words with sequences of high front vowels, items with clusters of interdentals and /s/ and forms which are subject to irregular morphological alternations. The next major source of errors is the existence of phonetic 'false friends', whose number runs into hundreds, not only in Polish, but also in many other languages as well. We would like to suggest that in phonetic practice use should be made of such 'minimal pairs,' employed in, for instance, Szpyra-Kozłowska and Sobkowiak (2011). They should include both common and proper nouns, e.g.

\begin{tabular}{llll} 
English & Polish & English & Polish \\
\hline atom & atom & Adam & Adam \\
boa & boa & Nepal & Nepal \\
safari & safari & Madrid & Madryt \\
marketing & marketing & Cameron & Cameron \\
model & model & Clinton & Clinton
\end{tabular}

\subsection{A comparison of word difficulty for intermediate and advanced learners}

A comparison of factors contributing to the difficulty of word pronunciation for intermediate and advanced learners shows that, statistically, the latter group has learnt to deal better with the issue of liquids, word stress, long words and spelling-related problems. Advanced learners have also fewer problems with consonantal clusters involving interdentals, with the exception of 'th' followed by $/ \mathrm{s} /$. The most difficult items turned out to be lengths and strengths, both containing clusters of three consonants. The issues which are problematic for both groups involve sequences of high front vowels within single words, phonetic 'false friends' and forms displaying irregular morphological alternations. This means that these difficulties should be given special attention in the phonetic training of all learners. These observations are summarized below. 


\section{Hierarchy of word pronunciation difficulty factors Intermediate learners}

\author{
All factors of similar \\ difficulty
}

\section{Advanced learners}

Most difficult:

high front vowels

$\langle$ th $+\mathrm{s}$ >

phonetic 'false friends'

less difficult:

morphological alternations

spelling-related problems

long words

stress-related problems

sequences of liquids

\subsection{Difficult and easy words}

Let us now examine in some detail those words which, among the 80 diagnostic lexical items, proved to be particularly difficult or easy for the participants. The easiest words to pronounce, with over $85 \%$ of proper realizations are the following:
(a) rural literally burglary barely
(b) monthly birthday hundredth
(c) variety (various) anxiety (anxious) sincerity (sincere)

The examples in (a) contain sequences of liquids while those in (b) clusters in which the interdentals are combined with nonfricatives. The words in (c) participate in morphological alternations, as seen in related forms provided in parentheses.

The most problematic items can also be divided into several sets.
(a) cheating ceiling greedy repeating deceiving
(b) courteous (court) advantageous (advantage) managerial (manager)
(c) algebra (P algebra) caricature (P karykatura)
(d) strengths lengths

The first and the largest of them in (a) contains sequences of high front vowels The next one in (b) involves irregular morphological alternations. The third group in (c) has cognates in Polish. Finally, the last one in (d) comprises clusters of velar nasals, followed by interdentals and /s/.

Moreover, some words might be claimed to cause difficulty because of the complex relationship between spelling and pronunciation, for instance those with the suffix -ous added to stems ending in 〈e〉, e.g. courteous hideous advantageous

It should also be noted that some of the most problematic items are fairly long and contain at least four syllables: advantageous caricature managerial

To sum up, the items provided in this section support the observations made earlier concerning the major sources of word mispronunciations. Yet another question that naturally arises in connection with the experimental items concerns the relationship between the phonetic difficulty of these words for the learners and their frequency of occurrence. We have found no meaningful relationship between these two issues. Thus, 
the frequency figures, based on the British National Corpus of Spoken English, are identical or almost identical for many words belonging both to category of difficult and easy words, e.g.

\section{Frequency of easy words \\ hundredth 2 \\ burglary 3 \\ sincerity 1}

\section{Frequency of difficult words courteous 2 \\ haven 3 \\ managerial 1}

The problem is that word frequency in the British National Corpus of Spoken English does not have to be the same as word frequency in foreign students' English for which no data are available. This means that the former source is of limited usefulness in predicting the degree of difficulty involved in word pronunciation.

\subsection{Experimental results versus Phonetic Difficulty Index}

In this section we examine the accuracy of Sobkowiak's Phonetic Difficulty Index, with its 10-point scale, in predicting the degree of difficulty of the experimental items.

It appears that in some cases the PDI values do coincide with the easy/difficult dichotomy established in this study, e.g. ${ }^{1}$
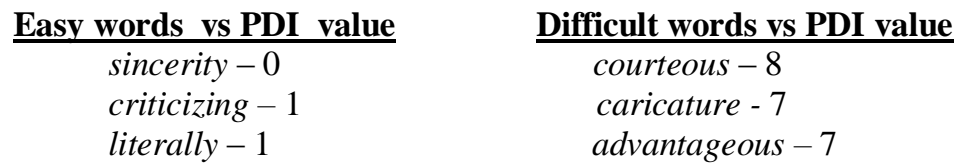

In the majority of cases, however, no significant correlation between the two evaluations can be found. Thus, frequently our easy and difficult words are given the same PDI values.

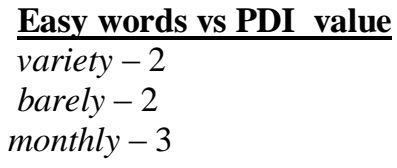

\author{
Difficult words vs PDI value \\ haven -2$$
\text { ceiling }-2
$$$$
\text { cheating }-3
$$

In some instances easy words have a higher PDI value than the difficult ones, e.g.

\section{Easy words vs PDI value}

$$
\begin{aligned}
& \text { rural }-4 \\
& \text { burglary }-5 \\
& \text { hundredth }-5
\end{aligned}
$$

\author{
Difficult words vs PDI value \\ strengths -3 \\ receiving -2 \\ algebra -3
}

\footnotetext{
${ }^{1}$ I am grateful to W. Sobkowiak for providing me with the PDI values of the experimental items. The difficulty scale ranges from 0 to 10 , where the higher the score, the greater the phonetic difficulty of words.
} 
An analysis of 24 easy and difficult experimental words with their PDI values shows that a correlation is found in about $50 \%$ of cases only. We can conclude that the PDI, in its present shape, is rather inaccurate as a measure of the phonetic difficulty of words for the advanced learners who took part in our experiment.

\subsection{Students' evaluation of word difficulty}

In the second part of the experiment the participants were asked to evaluate the degree of difficulty involved in the pronunciation of 24 experimental items representing eight types of factors isolated in section 2.

According to the subjects, the following factors make words difficult for them to pronounce:

- length (e.g. unintelligibility, satisfactorily)

- low frequency of occurrence (e.g. unintelligibility, satisfactorily)

- the presence of $<\mathrm{th}+\mathrm{s}>$ clusters (e.g. sixths, lengths, maths)

- spelling and specific word endings (such as -eous) (e.g. thouroughly, courteous, southern)

Thus, the most frequently mentioned source of word difficulty was their considerable length (left unspecified), with two words judged as the most problematic of the tested items, i.e. unintelligibility and satisfactorily. The low frequency of the same words was also indicated as a cause of pronunciation problems. Sixth, lengths and maths, all containing interdental fricatives followed by /s/, were listed as difficult because of such clusters. Irregular spelling and sound correspondences in thouroughly, courteous, southern and anxiety were blamed for pronunciation problems with these items. The participants also enumerated some troublesome word endings, e.g. - eous (courteous), rily (satisfactorily), -rely (rarely). Interestingly, problems with the correct placement of stress were not mentioned.

Of the 24 items subject to students' evaluation, the following five were judged the most difficult (the figures in parentheses indicate the number of participants who evaluated these words as such): unintelligibility (15) satisfactorily (10) courteous (5) thoroughly (5) sixth (5)

It is interesting to examine whether these judgements find confirmation in the students' actual performance. Taking into account the 24 items under consideration, the most difficult words for them to pronounce were as follows (with the percentage of correct realizations provided in parentheses): courteous $(0 \%)$ greedy $(0 \%)$ innocent $(8 \%)$ ceiling $(8 \%)$ thoroughly $(16 \%)$

These data show that the participants' opinions on word difficulty coincide with their phonetic performance only in the case of two items, i.e. courteous and thoroughly. In the remaining instances there is no such correlation. Thus, two words claimed to be the most difficult received the following scores: unintelligibility was pronounced correctly by $30 \%$ of the students and satisfactorily by $58 \%$, which makes them items of medium difficulty. Interestingly, none of the respondents mentioned as problematic the following words with fairly low scores for correctness: Murphy (20\%) lengths (20\%) southerners $(25 \%)$ Nepal $(25 \%)$ 
The observations reported here indicate that even advanced learners who undergo formal phonetic training are only partly aware of their pronunciation problems.

Let us examine in more detail the relationship between the students' phonetic performance and their assessment of word difficulty. We compared 8 evaluations of 24 words as easy or difficult to pronounce by 4 students with good pronunciation and 4 students with poor pronunciation with their actual realization of these items. We counted the number of matches and mismatches between the questionnaire answers ${ }^{2}$ and the participants ' production of a given item. The results are shown below.

\begin{tabular}{lcc} 
& $\begin{array}{c}\text { items judged easy } \\
\text { and pronounced correctly }\end{array}$ & $\begin{array}{c}\text { items judged easy } \\
\text { and mispronounced }\end{array}$ \\
\cline { 2 - 3 } good students & 26 & 11 \\
poor students & 9 & 24
\end{tabular}

It turned out that good students were more accurate in their assessment of word difficulty than poor students. This means that good students more often consider words as easy when they can actually pronounce them correctly than poor students who often mark words as easy and yet mispronounce them.

\section{Conclusions}

It is hoped that the presented study has provided some insight into the issue of phonetically difficult words in the speech of advanced Polish learners. It has allowed us to make several observations which carry important pedagogical implications.

1. Phonetically difficult words abound in Polish-accented English of learners of different levels of proficiency, including intermediate and advanced students. Consequently, this issue should be given due attention in the course of their phonetic training.

2. The most important sources of word mispronunciations for advanced learners involve sequences of high front vowels, clusters of interdentals with other fricatives and phonetic 'false friends.'

3. Advanced students, when compared with intermediate learners, have fewer problems with spelling and stress-related issues, sequences of liquids, longer words, and clusters of interdentals with nonfricatives.

4. Since sets of phonetically difficult words for intermediate and advanced learners overlap only partially, there can be no one PDI common for all of them.

5. The comparison of students' evaluation of word difficulty with the experimental results indicates that even advanced learners are only partly aware of their pronunciation problems and cannot assess them objectively. Thus, more care should be taken to develop their skill of self-evaluation.

\footnotetext{
${ }^{2}$ A match was declared when an item was pronounced correctly and marked as easy to pronounce or when an item was mispronounced and marked as difficult to pronounce.
} 


\section{References}

Sobkowiak, W. 1999. Pronunciation in EFL Machine-Readable Dictionaries. Poznań: Motivex.

Szpyra-Kozłowska, J. 2011. Phonetically difficult words in intermediate learners' English. In Pawlak, M,. E. Waniek-Klimczak \& J. Majer (eds). Speaking in contexts of instructed foreign language acquisition.. Bristol: Multilingual Matters. 286-299.

Szpyra-Kozłowska, J. \& W. Sobkowiak. 2011. Workbook in English Phonetics. $2^{\text {nd }}$ edition. Lublin: Wydawnictwo UMCS.

Szpyra-Kozłowska, J \& S. Stasiak. 2010. From focus on sounds to focus on words in English pronunciation instruction. Research in Language vol. 8., 163-174.

Szpyra-Kozłowska, J. in press On the irrelevance of sounds and prosody in foreignaccented English.

Waniek-Klimczak, E. 2002. Akcent wyrazowy w nauczaniu języka angielskiego. In Sobkowiak, W. \& E. Waniek-Klimczak (eds) Dydaktyka Fonetyki Języka Obcego. Zeszyty Naukowe PWSZ w Płocku, t. III. Płock: Wydawnictwo Naukowe PWSZ w Płocku. 101-114. 


\section{Appendix 1}

A list of diagnostic sentences. The tested items are in boldface.

1. His mania for watching Disney cartoons and horror films all night made the hotel management increasingly uncomfortable.

2. Graham and Murphy went straight from Madrid to Nepal, where they joined the demonstrators fighting for freedom in Tibet.

3. It is frequently claimed that at school Einstein showed no enthusiasm or appreciation for algebra, and his maths teacher regularly accused him of cheating.

4. He went to great lengths to characterize appropriately all his strengths and weaknesses to prove he was innocent and did not commit this burglary.

5. With mounting curiosity he examined the whole area thoroughly for the sixth time and decided that the evidence that the infamous murderer was there was not satisfactorily established and was purely circumstantial.

6. The artificiality and unintelligibility of his explanations created much anxiety particularly in this rural area were politicians are rarely trusted and their sincerity is frequently questioned.

7. The rivalry between the southerners and the northerners in this sleepy town was closely watched by the managerial staff of this industry who thought it advantageous for a variety of reasons.

8. In this monthly I saw a caricature of this admirable, courteous man whom the media keep simultaneously praising and criticizing.

9. It was her hundredth birthday and the organizers of the party made every effort to control the chaos and provide various attractions: a gigantic neon with a congratulatory message, a champagne geyser and a band of robots playing very rhythmical music.

10. They considered this place their ultimate haven, with chestnut trees, thyme and heather in the garden decorated with hideous dwarfs.

11. This greedy, unsophisticated person who kept repeating that he was capable of deceiving literally everybody held a prestigious administrative post.

12. He could barely wait for receiving an explanatory statement from this authoritarian official.

13. The width of this ceiling was truly impressive, but the paintings on it were fairly imitative. 


\section{Appendix 2}

The questionnaire used in the experiment

Evaluate the difficulty of pronunciation of the following words by marking them as

E - easy to pronounce

M-medium

D - difficult to pronounce

1. thoroughly

3. lengths

5. administrative -

2. demonstrators

7. greedy

4. Murphy

9. curiosity

6. particularly

11. sixth

8. anxiety

10. geyser

13. burglary

12. unintelligibility

15 . southern

14. ceiling

17. chestnut

16. explanatory

19. criticizing

21. rarely

18. Nepal

20. satisfactorily

23. innocent

22. courteous

24. maths

Now choose three words from the above list which you consider difficult to pronounce and comment on why you find them difficult. 